01

\title{
Электростатическое трение зонда атомно-силового микроскопа при движении у поверхности образца
}

\author{
(C) Г.В. Дедков, А.А. Канаметов \\ Кабардино-Балкарский государственный университет, \\ 360004 Нальчик, Россия \\ e-mail: gv_dedkov@mail.ru, kanametov_a@mail.ru
}

(Поступило в Редакцию 18 сентября 2017 г. В окончательной редакции 20 декабря 2017 г.)

В рамках решения нерелятивистской электродинамической задачи получены общие формулы для тангенциальной диссипативной силы электростатического трения и нормальной силы притяжения к поверхности аксиально-симметричного зонда, движущегося параллельно гладкой поверхности однородных материалов, или покрытых тонкими пленками подложек с различным сочетанием материалов. В качестве численного примера рассмотрено взаимодействие металлического шарика, движущегося над металлической поверхностью. Результаты расчета сравниваются с имеющимися экспериментальными и теоретическими результатами других авторов.

DOI: 10.21883/JTF.2018.11.46619.2497

\section{Введение}

Электростатическая сила трения является одной из возможных составляющих диссипативных сил между двумя телами, разделенных вакуумным или воздушным зазором, при их относительном движении. Статическое электрическое поле между поверхностями может существовать даже без внешнего напряжения. В частности, электростатическое притяжение при нулевом напряжении на контакте может быть вызвано локальными изменениями работы выхода или поверхностными загрязнениями, приводящими к неоднородному электрическому полю (вследствие эффекта зарядовых пятен) [1-3]. Кроме того, пространственные флуктуации электрического поля могут быть вызваны заряженными дефектами в объеме зонда или образца.

Электростатические силы между проводящими поверхностями, возникающие из-за изменений поверхностного потенциала, изучались в [3]. Изменения потенциала, обусловленного пятнами заряда, измерялись в различных условиях с использованием вибрационных или вращающихся пластинчатых электрометров [4]. Ряд работ был посвящен электростатическим силам в атомносиловой микроскопии (АСМ) (см., например, [5-7]). Измерения электростатических сил имеют большое значение не только для интерпретации данных АCM, но и для точных измерений сил Казимира [8], а также при проектировании МЭМС [9,10].

Гораздо меньше внимания уделялось проблеме диссипативных электростатических сил при движении зондирующих тел вблизи поверхности [11-15]. В частности, теоретические модели электростатических сил трения обсуждались в $[12,15]$. В этих работах кончик металлического зонда рассматривался как цилиндрический наконечник с радиусом $R$. Предполагалось, что наконечник движется параллельно поверхности. Кроме того, авторы также рассмотрели модель сферического [12,15] и эллипсоидального наконечника [12], пренебрегая пространственными изменениями потенциала из-за его кривизны [15], или используя локально-плоское приближение [12]. Наряду с этим использовались упрощенные выражения для емкости контакта зонд-образец [15]. Коэффициент электростатического трения вычислялся, исходя из джоулевых потерь в объеме образца [12], или через вектор Пойнтинга на поверхности образца [15]. Оба метода привели к близким численным оценкам силы электростатического трения и сходству зависимостей сил трения от расстояния до поверхности и от радиуса наконечника. Однако численные значения сил трения оказались значительно меньше, чем значения диссипативных сил в эксперименте [11].

Целью настоящей работы является прямой расчет электростатической силы, приложенной к движущемуся зонду, имеющему коническую форму со сферическим окончанием, или произвольную аксиальносимметричную форму. Распределение зарядов на зонде считается фиксированным (в виде случайным образом распределенных пятен заряда) или контролируется внешним напряжением $U$ на контакте. Распределение заряда, индуцированного в образце, получается путем решения точной электродинамической задачи с учетом реальной формы наконечника и диэлектрических свойств образца. Результирующая сила трения, приложенная к зонду, рассчитывается методом, использованным при расчете силы трения, действующей на единичный точечный заряд, движущийся над поляризующейся поверхностью [16]. Поскольку зонд АСМ может иметь очень острую оконечность, плотность заряда максимальна вблизи его апекса и быстро уменьшается с увеличением расстояния от образца. Соответственно точное определение распределения заряда имеет решающее значение для корректного расчета силы трения. 


\section{1. Нормальные и тангенциальные электростатические силы, действующие на движущийся зонд с фиксированным распределением зарядов}

На рис. 1 показана конфигурация системы и используемая декартова система координат $(x, y, z)$, связанная с образцом. Пробное тело с фиксированными точечными зарядами $q_{j}$, имеющими координаты $\left(x_{j}+V t, y_{j}, z_{j}\right)$, движется с постоянной скоростью $V$ над образцом с диэлектрической проницаемостью , зависящей от частоты $\varepsilon(\omega)$. В более общем случае (см. вставку), образец представляет собой толстую подложку и пленку с толщиной $d$, характеризующиеся диэлектрическими проницаемостями $\varepsilon_{2}(\omega)$ (пленка) и $\varepsilon_{3}(\omega)$ (подложка). В рамках нерелятивистского приближения $V / c \ll 1$ электродинамическая задача сводится к решению уравнения Пуассона для электростатического потенциала $\phi(x, y, z, t)$ :

$$
\begin{aligned}
& \Delta \phi(x, y, z, t)=-4 \pi \rho(x, y, z, t) \\
& \quad=-4 \pi \sum_{j} q_{j} \delta\left(x-\left(x_{j}+V t\right)\right) \delta\left(y-y_{j}\right) \delta\left(z-z_{j}\right) .
\end{aligned}
$$

Уравнение (1) должно решаться с граничными условиями непрерывности потенциала $\phi$ и нормальной проекции электрического смещения $-\varepsilon d \phi / d z$ при $z=0$ в случае однородного образца и при $z=0, z=-d$ в случае подложки, покрытой пленкой. Записывая фурье-разложения для потенциала $\phi(\mathbf{r}, z, t)$ и плотности распределения заряда $\rho(\mathbf{r}, z, t)$ в правой части $(1)$, где $\mathbf{r}-$ двумерный вектор в плоскости образца $(x, y), \mathbf{k}=\left(k_{x}, k_{y}\right)$, будем



Pис. 1. Геометрическая конфигурация и декартова система координат, используемая в расчетах. иметь

$$
\begin{aligned}
& \phi(\mathbf{r}, z, t)=\frac{1}{(2 \pi)^{3}} \int d^{2} k d \omega \phi_{\omega \mathbf{k}}(z) \exp [i(\mathbf{k r}-\omega t)], \\
& \rho(\mathbf{r}, z, t)=\frac{1}{(2 \pi)^{3}} \int d^{2} k d \omega \rho_{\omega \mathbf{k}}(z) \exp [i(\mathbf{k r}-\omega t)] .
\end{aligned}
$$

Подставляя (2), (3) в (1), получим уравнение

$$
\left(\frac{d^{2}}{d z^{2}}-k^{2}\right) \phi_{\omega \mathbf{k}}(z)=-8 \pi^{2} \delta\left(\omega-k_{x} V\right) \sum_{j} q_{j} \exp \left(-i \mathbf{k r}_{j}\right) .
$$

Решая (4) с указанными граничными условиями (при $z=0$ и $z=-d)$, получим

$$
\begin{gathered}
\phi_{\omega \mathbf{k}}(z)=\phi_{\omega \mathbf{k}}^{\text {bare }}(z)+\phi_{\omega \mathbf{k}}^{i n d}(z), \\
\phi_{\omega \mathbf{k}}^{\text {bare }}(z)=-\frac{4 \pi^{2}}{k} \delta\left(\omega-k_{x} V\right) \sum_{j} q_{j} \exp \left(-\mathbf{k} \mathbf{r}_{j}\right) \\
\times \exp \left(k\left|z-z_{j}\right|\right), \\
\phi_{\omega \mathbf{k}}^{i n d}=-\frac{4 \pi^{2}}{k} D(\omega, k) \delta\left(\omega-k_{x} V\right) \sum_{j} q_{j} \exp \left(-\mathbf{k r}_{j}\right) \\
\times \exp \left(-k\left(z+z_{j}\right)\right), \\
D(\omega, k)=\frac{\Delta_{1}(\omega)-\Delta_{2}(\omega) \exp (-2 k d)}{1-\Delta_{1}(\omega) \Delta_{2}(\omega) \exp (-2 k d)} \\
\Delta_{1}(\omega)=\frac{\varepsilon_{2}(\omega)-1}{\varepsilon_{2}(\omega)+1}, \Delta_{2}(\omega)=\frac{\varepsilon_{2}(\omega)-\varepsilon_{3}(\omega)}{\varepsilon_{2}(\omega)+\varepsilon_{3}(\omega)} .
\end{gathered}
$$

Фурье-образы $\phi_{\omega \mathbf{k}}^{\text {bare }}(z)$ и $\phi_{\omega \mathbf{k}}^{\text {ind }}(z)$ в $(5)-(7)$ соответствуют потенциалу зарядов зонда и индуцированному потенциалу образца. Подставляя (6), (7) в (2) и интегрируя по частоте $\omega$, получим

$$
\begin{aligned}
\phi^{\text {bare }}(\mathbf{r}, z, t) & =-\frac{1}{2 \pi} \sum_{j} q_{j} \int \frac{d^{2} k}{k} \exp \left(-k\left|z-z_{j}\right|\right) \\
& \times \exp \left(i \mathbf{k}\left(\mathbf{r}-\mathbf{r}_{j}\right)-i k_{x} V t\right), \\
\phi^{i n d}(\mathbf{r}, z, t) & =-\frac{1}{2 \pi} \sum_{j} q_{j} \iint \frac{d^{2} k}{k} D\left(k_{x} V, k\right) \\
& \times \exp \left(-k\left(z+z_{j}\right)\right) \exp \left(i \mathbf{k}\left(\mathbf{r}-\mathbf{r}_{j}\right)-i k_{x} V t\right) .
\end{aligned}
$$

Индуцированное электрическое поле получается из (2) и (11) с учетом уравнения $\mathbf{E}^{\text {ind }}=-\nabla \phi^{\text {ind }}$. Суммируя электростатические силы $q_{i} \mathbf{E}^{\text {ind }}\left(x=x_{j}+V t, y=y_{j}\right.$, $\left.z=z_{j}, t\right)$, приложенные к точечным зарядам зонда, получим выражения для тангенциальной и нормальной сил $F_{x}, F_{z}$, действующих на него:

$$
\begin{aligned}
F_{x} & =\frac{i}{2 \pi} \sum_{j} \sum_{m} q_{j} q_{m} \int d^{2} k \frac{k_{x}}{k} D\left(k_{x} V, k\right) \\
& \times \exp \left(-i \mathbf{k}\left(\mathbf{r}_{j}-\mathbf{r}_{m}\right)\right) \exp \left(-k\left(z_{j}+z_{m}\right)\right),
\end{aligned}
$$




$$
\begin{aligned}
F_{z} & =\frac{-1}{2 \pi} \sum_{j} \sum_{m} q_{j} q_{m} \int d^{2} k D\left(k_{x} V, k\right) \\
& \times \exp \left(-i \mathbf{k}\left(\mathbf{r}_{j}-\mathbf{r}_{m}\right)\right) \exp \left(-k\left(z_{j}+z_{m}\right)\right),
\end{aligned}
$$

где $\mathbf{r}_{m}=\left(x_{m}, y_{m}\right), \mathbf{r}_{j}=\left(x_{j}, y_{j}\right)$. Формулы (10)-(13) имеют общий характер независимо от формы зонда и распределения зарядов. В частности, для единичного движущегося заряда формулы $(10)-(13)$ сводятся к хорошо известным результатам [16].

Как следует из (12), (13), электростатические силы зависят от свойств функции диэлектрического отклика (8). Поэтому целесообразно рассмотреть несколько характерных комбинаций материалов зонда и образца. Сила трения $F_{x}$ определяется мнимой частью функции $D(\omega, k)$, а сила притяжения $F_{z}$ - вещественной частью $D(\omega, k)$. Характерные скорости зондов АСМ очень малы даже в динамических режимах сканирования АСМ $(V \ll 1 \mathrm{~m} / \mathrm{s})$, а при типичном расстоянии апекса зонда от поверхности $(10 \mathrm{~nm})$ основные вклады в интегралы в (10)-(13) связаны с волновыми векторами порядка $10^{8} \mathrm{~m}^{-1}$. Соответственно частоты $\omega=k_{x} V$ имеют порядок $10^{8} \mathrm{~s}^{-1}$. В этом случае мнимые части $D(\omega, k)$ и $\Delta_{1,2}(\omega)$ в $(6),(7)$ оказываются значительно меньше вещественных частей для любой типичной пары контактирующих материалов (металлов, полупроводников, ионных диэлектриков, водных пленок или их сочетаний). Поэтому с точностью порядка $\left(\Delta_{1,2}^{\prime \prime} / \Delta_{1,2}^{\prime}\right)^{2}$ из (6) получим

$$
\begin{aligned}
& \operatorname{Im}\left(D\left(k_{x} V, k\right)\right) \approx \frac{\Delta_{1}^{\prime}\left(1-a^{2}\left|\Delta_{2}\right|^{2}\right)+a \Delta_{2}^{\prime \prime}\left(\left|\Delta_{1}\right|^{2}-1\right)}{1+a^{2}\left|\Delta_{1}\right|^{2}\left|\Delta_{2}\right|^{2}-2 a \Delta_{1}^{\prime} \Delta_{2}^{\prime}}, \\
& \operatorname{Re}\left(D\left(k_{x} V, k\right)\right) \approx \frac{\left(\Delta_{1}^{\prime}-a \Delta_{2}^{\prime}\right)\left(1-a \Delta_{1}^{\prime} \Delta_{2}^{\prime}\right)}{\left(1+a^{2}\left|\Delta_{1}\right|^{2}\left|\Delta_{2}\right|^{2}-2 a \Delta_{1}^{\prime} \Delta_{2}^{\prime}\right)}
\end{aligned}
$$

где $a=\exp (-2 k d)$, а величины с одним и двумя штрихами обозначают вещественную и мнимую части функций $\Delta_{1,2}$.

В соответствии с принятым низкочастотным приближением диэлектрические проницаемости металлов и ионных диэлектриков можно записать в виде

$$
\begin{gathered}
\varepsilon(\omega)=1+i \frac{4 \pi \sigma}{\omega}, \\
\varepsilon(\omega)=\varepsilon_{\infty}+\frac{\left(\varepsilon_{0}-\varepsilon_{\infty}\right) \omega_{T}^{2}}{\omega_{T}^{2}-\omega^{2}-i \gamma \omega} \approx \varepsilon_{\infty}+i \frac{\left(\varepsilon_{0}-\varepsilon_{\infty}\right) \gamma \omega}{\omega_{T}^{2}},
\end{gathered}
$$

где $\sigma$ - проводимость металла, $\varepsilon_{0}, \varepsilon_{\infty}-$ статическая и оптическая диэлектрические проницаемости диэлектрика, $\gamma$ и $\omega_{T}-$ фактор затухания и частота поперечных фононов. Выражение, аналогичное (16), (17), можно также использовать для диэлектриков с вращательными релаксациями. Например, для воды можно использовать приближение

$$
\varepsilon(\omega)=\alpha+\frac{\beta}{1+i \omega / \omega_{0}}
$$

с параметрами $\alpha=4.35, \beta=72.24, \omega_{0}=1.3 \cdot 10^{11} \mathrm{~s}^{-1}$.
Используя (8), (9) (14)-(18), легко получить выражения для $\operatorname{Im}\left(D\left(k_{x} V, k\right)\right)$ и $\operatorname{Re}\left(D\left(k_{x} V, k\right)\right)$ в случае чистых поверхностей с диэлектрическими функциями (16)-(18) или с параметрами, соответствующими другим сочетаниям материалов зонда и поверхности. Соответствующие формулы приведены в Приложении и могут применяться при условии $\omega_{0} z / V \gg 1$, где $\omega_{0}$ - характерная частота поглощения $\left(2 \pi \sigma, \omega_{T}\right.$ или $\omega_{0}$ в случае функций $(16)-(18)), z$ - характерное расстояние между зондом и образцом.

\section{2. Распределение заряда и электростатические силы при наличии напряжения между зондом и образцом}

Пусть проводящий зонд нагружается внешним напряжением $U$ и перемещается параллельно заземленному образцу, представляющему однородную металлическую пластину, металлическую пленку поверх диэлектрической пластины, или диэлектрическую пленку поверх металлической пластины. Общее решение уравнения Пуассона определяется суммой (10) и (11) при условии $\phi_{S}=U$ на поверхности зонда. Сами заряды зонда пока считаются неизвестными. При подстановке мгновенных координат $(\mathbf{r}, z)=(x+V t, y, z)$ любой точки зонда в (10) и (11) можно видеть, что результирующий потенциал $\phi_{S}$ не зависит от времени. Поэтому распределение зарядов на зонде тоже не зависит от времени. Однако индуцированный в образце потенциал зависит от времени и пространственных координат.

В практически важном случае аксиально-симметричного проводящего зонда (наконечника АCM, рис. 2) заряд распределен на поверхности, и его распределение также должно быть аксиально-симметричным. Используя этот факт, заменим дискретные заряды $q_{m}, q_{j}$ в (8)-(13) кольцевыми зарядами $q_{m}, q_{j}$, дополнительно разбивая их на дискретные заряд $q_{m n}$ и $q_{j p}$, соответствующие кольцам $m$ и $j$. Учитывая аксиальную симметрию, заменим дополнительное суммирование по индексам $n$ и $p$ угловым интегрированием. В результате, используя аналитические свойства $D(\omega, k)$ (см. Приложение), для точек $(r, z) \in S$ на поверхности зонда формулы (10)-(13) принимают вид

$$
\begin{aligned}
\phi^{\text {bare }}(r, z) & =\sum_{j} q_{i} \int_{0}^{\infty} d k J_{0}(k r) J_{0}\left(k r_{j}\right) \exp \left(-k\left|z-z_{j}\right|\right) \\
= & \sum_{j} q_{j} T\left(r, r_{j},\left|z-z_{j}\right|\right) \\
T(x, y, z) & =\frac{2}{\pi} \frac{1}{\pi\left[z^{2}+(x+y)^{2}\right]^{1 / 2}} \\
& \times K\left[\left(\frac{4 x y}{\left[z^{2}+(x+y)^{2}\right]}\right)^{1 / 2}\right]
\end{aligned}
$$




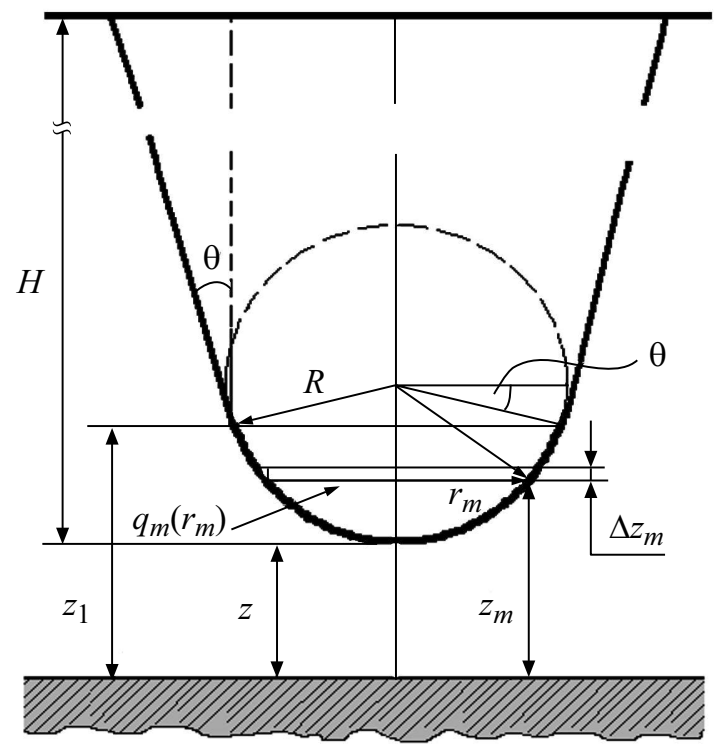

Pис. 2. Схематический вид конического зонда со сферическим окончанием, характеризующимся параметрами $R, H, \theta$; $r_{m}$ - радиус $m$-го кольца поверхности зонда с зарядом $q_{m}$, $\Delta z_{m}$ - шаг сетки, соответствующий кольцу с номером $m$, $z_{1}$ - высота точки на поверхности зонда, в которой сферическая оконечность сопрягается с конической.

$$
\begin{aligned}
\phi^{i n d}(r, z)= & -\frac{1}{2 \pi} \sum_{j} q_{j} \int \frac{d^{2} k}{k} D\left(k_{x} V, k\right) J_{0}(k r) J_{0}\left(k r_{j}\right) \\
& \times \exp \left(-k\left(z+z_{j}\right)\right), \\
F_{x}= & -\frac{1}{2 \pi} \sum_{m} \sum_{j} q_{m} q_{j} \int d^{2} k \frac{k_{x}}{k} \operatorname{Im}\left(D\left(k_{x} V, k\right)\right) J_{0}\left(k r_{m}\right) \\
& \times J_{0}\left(k r_{j}\right) \exp \left(-k\left(z_{m}+z_{j}\right)\right), \\
F_{z}= & -\frac{1}{2 \pi} \sum_{m} \sum_{j} q_{m} q_{j} \int d^{2} k \operatorname{Re}\left(D\left(k_{x} V, k\right)\right) J_{0\left(k r_{m}\right)} J_{0}\left(k r_{j}\right) \\
\quad & \exp \left(-k\left(z_{m}+z_{j}\right)\right)
\end{aligned}
$$

В (19)-(23) $K(x)$ - эллиптический интеграл первого рода, а $J_{0}(x)$ - функция Бесселя. Разлагая $D\left(k_{x} v, k\right)$ (см. Приложение) по степеням $\left(k_{x} V\right)$ и подставляя в $(21)$, легко найти, что с точностью до членов порядка $V^{2}$ индуцированный потенциал поверхности имеет вид

$$
\begin{aligned}
\phi^{i n d}(r, z) \approx & -\sum_{j} q_{j} \int_{0}^{\infty} d k \operatorname{Re}(D(0, k)) J_{0}(k r) J_{0}\left(k r_{j}\right) \\
& \times \exp \left(-k\left(z+z_{j}\right)\right) .
\end{aligned}
$$

Таким образом, в приближении малых скоростей индуцированный потенциал зонда совпадает с потенциалом индуцированных зарядов, соответствующим статической электростатической задаче. Сила притяжения зонда к образцу в этом приближении также не зависит от его скорости. В отличие от этого тангенциальная сила $F_{x}$ зависит от временной задержки между движущимся зарядом и индуцированным полем образца. Это приводит к силе трения, которая линейно зависит от скорости зонда $V$.

В соответствии с результатами, приведенными в Приложении:

$$
\begin{aligned}
& \operatorname{Re}(D(0, k))=A \frac{p+\exp (-2 k d)}{1+p \exp (-2 k d)} \\
& =A \sum_{s=1}^{\infty}(-1)^{s-1} p^{s-1}[p \exp (-2 k d(s-1))+\exp (-2 k d n)],
\end{aligned}
$$

где $p$ - параметр (для чистых поверхностей однородных материалов, в частности $d \rightarrow \infty$ и $\operatorname{Re}(D(0, k))=$ $=p=\mathrm{const}$ ). Подставляя (25) в (24), получим

$$
\begin{aligned}
& \phi^{i n d}(r, z)=-p \sum_{j} q_{j} \sum_{s=1}^{\infty}(-p)^{s-1} \\
& \times\left[p T\left(r, r_{j}, 2 d(s-1)+z+z_{j}\right)+T\left(r, r_{j}, 2 d s+z+z_{j}\right)\right] .
\end{aligned}
$$

Следуя [17], для определения неизвестных зарядов на зонде используем метод наименьших квадратов с минимизирующей функцией $\sum_{n}\left(\phi_{S}-U\right)^{2}=\min , 1 \leq n \leq N$, где $\phi_{S}=\phi^{\text {bare }}\left(r_{n}, z_{n}\right)+\phi^{\text {ind }}\left(r_{n}, z_{n}\right)$. Дискретные точки на поверхности зонда $S$ с координатами $r\left(z_{n}\right), z_{n}$ выбираются в соответствии с уравнением $r(z)$ поверхности. Дискретная сетка $z_{m}$ вдоль оси $z$ для кольцевых зарядовых распределений строится таким образом, чтобы точки $z_{n}$ были расположены посередине между узлами $z_{m}(1 \leq m \leq N-1)$. Получаемая система линейных уравнений для кольцевых зарядов решается стандартными методами.

Зная кольцевые заряды $q_{j}$ и записывая $D\left(k_{x} V, k\right)$ в виде

$$
D\left(k_{x} V, k\right) \cong f_{1}(k)+i k_{x} V f_{2}(k),
$$

где функции $f_{1,2}(k)$ зависят от материальных свойств образца (см. Приложение), формулы (22) и (23) можно переписать в виде, более удобном для численных расчетов:

$$
\begin{gathered}
F_{x}=-\frac{V}{2} \sum_{m} \sum_{j} q_{m} q_{j} \int_{0}^{\infty} d k k^{2} f_{2}(k) \\
\times J_{0}\left(k r_{m}\right) J_{0}\left(k r_{j}\right) \exp \left(-k\left(z_{m}+z_{j}\right)\right), \\
F_{z}=-\sum_{m} \sum_{j} q_{m} q_{j} \int d k k f_{1}(k) J_{0}\left(k r_{m}\right) J_{0}\left(k r_{j}\right) \\
\times \exp \left(-k\left(z_{m}+z_{j}\right)\right)
\end{gathered}
$$

\section{3. Результаты численного расчета}

В качестве важного численного примера рассмотрим результаты расчета электростатических сил $F_{x}, F_{z}$ действующих на сферический металлический зонд (шарик) 
с радиусом $R$, движущийся параллельно поверхности металлической пластины со скоростью $V$. Потенциалы поверхности зонда и поверхности положим равными $U$ и нулю соответственно. Для диэлектрической проницаемости обоих материалов используем формулу (16). В этом случае в (27) и (28) $f_{1}=1, f_{2}=1 / 2 \pi \sigma$.

Общее число кольцевых зарядов принималось равным $N=300$. Шаг сетки по вертикальной координате возрастал с увеличением $z_{m}$ в геометрической прогрессии таким образом, что отношение максимального и минимального значений шага было близко к 20.

Для дальнейшего упрощения формулы (27) и (28) приводились к более простому виду после введения безразмерных величин зарядов $\tilde{q}_{m}=q_{m} / U R$ и переменной $u=z / R$ :

$$
\begin{gathered}
F_{x}=-\frac{V U^{2}}{2 R} f_{0} \sum_{m, j} \tilde{q}_{m} \tilde{q}_{j} T_{2}\left(r_{m} / R, r_{j} / R,\left(z_{m}+z_{j}\right) / R\right), \\
F_{z}=U^{2} \sum_{m, j} \tilde{q}_{m} \tilde{q}_{j} T_{1}\left(r_{m} / R, r_{j} / R,\left(z_{m}+z_{j}\right) / R\right),
\end{gathered}
$$

где вспомогательные функции $T_{1,2}(x, y, z)$ определены соотношениями

$$
\begin{gathered}
T_{1}(x, y, z)=\int_{0}^{\infty} d k k J_{0}(k x) J_{0}(k y) \exp (-k z)=\frac{d}{d z} T(x, y, z) \\
T_{2}(x, y, z) \equiv \int_{0}^{\infty} d k k^{2} J_{0}(k x) J_{0}(k y) \\
\times \exp (-k z)=\frac{d^{2}}{d z^{2}} T(x, y, z)
\end{gathered}
$$

Для функции $K(x)$ в (20) применялась аппроксимация [18]

$$
\begin{gathered}
K(x)=\int_{0}^{\pi / 2} \frac{d y}{\left(1-x^{2} \sin ^{2} y\right)^{1 / 2}} \approx \sum_{n=0}^{4}\left(a_{n} \eta^{n}-b_{n} \eta^{n} \ln \eta\right) \\
\eta=1-x^{2}
\end{gathered}
$$

Полиномиальное разложение (33) имеет погрешность, не превышающую $2 \cdot 10^{-8}$ (коэффициенты $a_{n}, b_{n}$ приведены в таблице Приложения).

На рис. 3 показаны рассчитанные значения кольцевых зарядов на шарике в относительных единицах $q_{n} / R U$ в зависимости от номера $n$ колец. Кривые $1-3$ соответствуют относительным расстояниям апекса шарика от поверхности $z_{0} / R=0.01,0.05,0.1$. Кажущееся увеличение плотности заряда вблизи наиболее удаленных от поверхности точек шарика (при $n \approx 300$ ) объясняется возрастанием площади колец из-за неравномерности сетки по высоте. Наибольшие изменения в распределении заряда происходят вблизи апекса шарика, обращенного к поверхности, в то время как в наиболее удаленных от

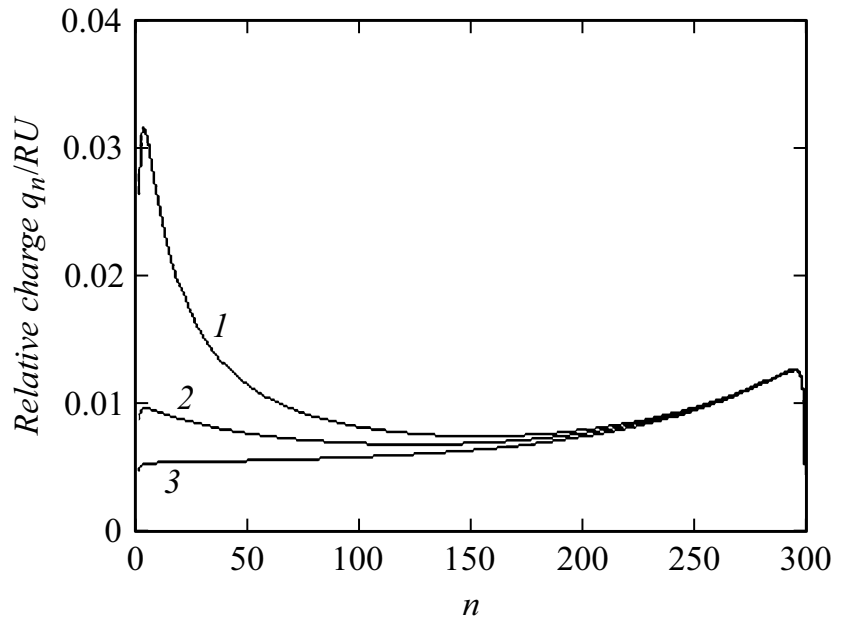

Рис. 3. Распределение кольцевых зарядов на поверхности металлического шарика. Линии 1-3 соответствуют расстояниям апекса шарика от поверхности $0.01,0.05$ и 0.1 (в единицах радиуса $R$ ). Частота кольцевых распределений прогрессивно уменьшается с ростом $n$ (в 20 раз) по мере движения от апекса к антиапексу шарика.

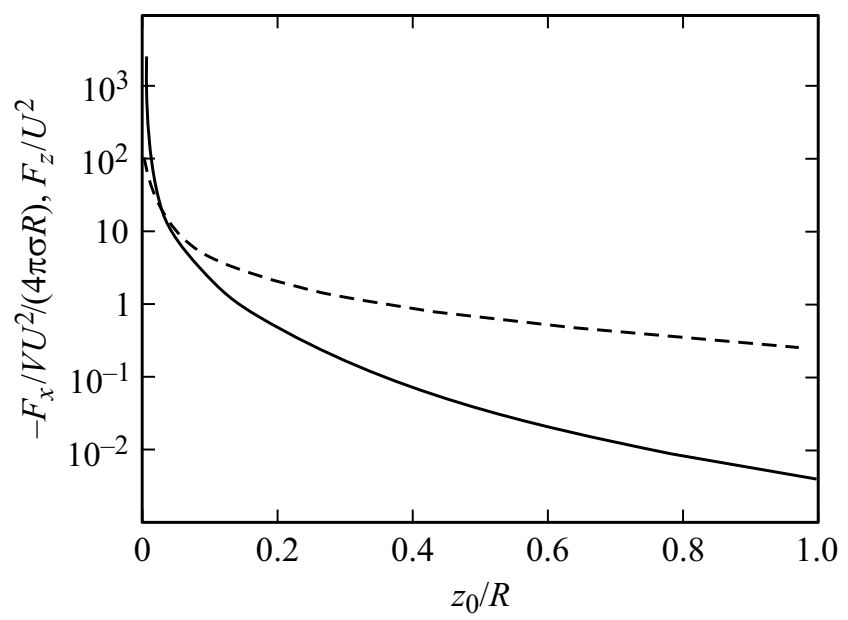

Рис. 4. Силы $F_{x}$ (сплошная линия) и $F_{z}$ (штриховая линия), выраженные в относительных единицах, в зависимости от расстояния апекса шарика от поверхности пластины. Для получения абсолютных значений сил (в динах) параметры $V$, $U, R$ и $\sigma$ должны быть выражены в гауссовых единицах.

поверхности точках шарика изменения в распределении заряда слабо зависят от минимального расстояния $z_{0}$ до поверхности.

На рис. 4 показаны результаты расчета сил $F_{x}$ и $F_{z}$ в относительных единицах $V U^{2} / 4 \pi \sigma R$ и $U^{2}$ в зависимости от относительного расстояния апекса $z_{0} / R$. В этом случае единичной силе $\tilde{F}_{x}$ соответствует абсолютное значение силы трения $0.478 \cdot 10^{-10} \mathrm{nN}$ (при $V=1 \mathrm{~m} / \mathrm{s}$, $U=1 \mathrm{~V}, R=10 \mathrm{~nm}, \sigma=1.85 \cdot 10^{17} \mathrm{~s}^{-1}$ для $\left.\mathrm{Au}\right)$, а единичной силе $\tilde{F}_{z}-$ абсолютное значение $1 / 9 \mathrm{nN}$. Заметим, что при выполнении расчетов значения $V, U$, $R$ и $\sigma$ в (29), (30) надо брать в гауссовых единицах. 
Пользуясь приведенными зависимостями (кривые 1 и 2), легко найти значения сил для любых расстояний $z_{0}$ и других параметров задачи.

Представляет интерес сравнить результаты расчета силы трения с экспериментом [11], в котором радиус сферического наконечника зонда $(\mathrm{Au})$ был равен $1 \mu \mathrm{m}$ (при тех же значениях остальных параметров). При $z_{0}=10 \mathrm{~nm}$ и $T=300 \mathrm{~K}$ измеренная величина диссипативной силы, пропорциональной скорости зонда, составляла $F_{x} \sim 1.5 \cdot 10^{-13} \mathrm{~N}$. В нашем случае, пользуясь графиком на рис. 4 , получим величину $F_{x} \sim 1.8 \cdot 10^{-20} \mathrm{~N}$, причем абсолютная зависимость от расстояния близка к $F_{x} \sim z_{0}^{-1.5}$ при $10 \leq z_{0} \leq 30 \mathrm{~nm}$ (как и в [11]). При меньших расстояниях от поверхности показатель степени $n$ в зависимости $F_{x} \sim z_{0}^{-n}$ ближе к 2. Абсолютное значение силы трения в несколько раз выше, чем следует из результатов расчета в $[15]\left(\sim 5.3 \cdot 10^{-21} \mathrm{~N}\right.$ при тех же значениях остальных параметров), но также значительно меньше, чем измеренная величина диссипативной силы. Таким образом, проблема интерпретации диссипативных сил в эксперименте [11] пока остается открытой.

\section{Заключение}

В рамках решения нерелятивистской электродинамической задачи получены общие формулы для тангенциальной диссипативной силы электростатического трения и нормальной силы притяжения к поверхности аксиально-симметричного зонда, движущегося параллельно поверхности однородных материалов, или покрытых тонкими пленками подложек с различными сочетаниями материалов. Показано, что результаты расчета силы трения сферического зонда для проводящих материалов зонда и поверхности можно представить в универсальной форме. Сравнение численных результатов с имеющимися экспериментальными значениями диссипативных сил в условиях электростатического взаимодействия обнаруживает расхождение на 8 порядков величины в меньшую сторону, как и в теоретических расчетах других авторов. Учитывая характер теоретической зависимости $F_{x}$ от материальных параметров, для экспериментального изучения электростатического трения, по-нашему мнению, целесообразнее использовать образцы (пластины) с низкой проводимостью (однородные, или с диэлектрическими покрытиями). Численный анализ сил электростатического трения для таких условий предполагается выполнить в следующих работах.

\section{Приложение}

Рассмотрим несколько типичных комбинаций материалов, используя уравнения (8), (9), (14)-(18). Соответствующие функции $\operatorname{Re}(D(\omega, k))$ и $\operatorname{Im}(D(\omega, k))$ имеют вид:
Чистые поверхности

а) металл

$$
\operatorname{Re} D=1, \operatorname{Im} D=\frac{\omega}{2 \pi \sigma}
$$

b) диэлектрик (17)

$$
\operatorname{Re} D=\frac{\varepsilon_{\infty}-1}{\varepsilon_{\infty}+1}, \operatorname{Im} D=\frac{2\left(\varepsilon_{0}-\varepsilon_{\infty}\right) \gamma \omega}{\left(\varepsilon_{\infty}+1\right)^{2} \omega_{T}^{2}}
$$

с) диэлектрик (18)

$$
\operatorname{Re} D=\frac{\alpha+\beta-1}{\alpha+\beta+1}, \operatorname{Im} D=\frac{2 \beta}{(\alpha+\beta+1)^{2}} \frac{\omega}{\omega_{0}} .
$$

Тонкие пленки с толщиной $d$ на толстой подложске а) металл (сверху)-диэлектрик (17) или (18) (снизу)

$$
\operatorname{Re} D=1, \operatorname{Im} D=\frac{1-\exp (-2 k d)}{1+\exp (-2 k d)} \frac{\omega}{2 \pi \sigma} ;
$$

b) диэлектрик (17) (сверху)-металл (снизу)

$$
\begin{aligned}
& \operatorname{Re} D=\frac{\exp (-2 k d)+\frac{\varepsilon_{\infty}-1}{\varepsilon_{\infty}+1}}{1+\exp (-2 k d) \frac{\varepsilon_{\infty}-1}{\varepsilon_{\infty}+1}}, \\
& \operatorname{Im} D=\frac{(1-\exp (-4 k d))}{\left(1+\exp (-2 k d) \frac{\varepsilon_{\infty}-1}{\varepsilon_{\infty}+1}\right)^{2}} \frac{2 \gamma \omega}{\omega_{r}^{2}}
\end{aligned}
$$

с) диэлектрик (18) (сверху)-металл (снизу)

$$
\begin{array}{r}
\operatorname{Re} D=\frac{\left(\frac{\alpha+\beta-1}{\alpha+\beta+1}+\exp (-2 k d)\right)}{\left(1+\exp (-2 k d) \frac{\alpha+\beta-1}{\alpha+\beta+1}\right)}, \\
\operatorname{Im} D=\frac{(1-\exp (-2 k d))}{(1+\exp (-2 k d))} \frac{2 \beta \omega}{(\alpha+\beta+1)^{2}} .
\end{array}
$$

Коэффициенты $a_{n}, b_{n}$ в (33) [18]

\begin{tabular}{c|c|c}
\hline$n$ & $a_{n}$ & $b_{n}$ \\
\hline 0 & 1.38629436112 & 0.5 \\
1 & 0.09666344259 & 0.12498593597 \\
2 & 0.03590092383 & 0.06880248576 \\
3 & 0.03742563713 & 0.03328355346 \\
4 & 0.01451196212 & 0.00441787012
\end{tabular}

\section{Список литературы}

[1] Young R.D., Clark H.E. // Phys. Rev. Lett. 1966. Vol. 17. P. 351.

[2] Burnham N.A., Colton R.J., Pollock H.M. // Phys. Rev. Lett. 1992. Vol. 69. P. 144. 
[3] Speake C.C., Trenkel C. // Phys. Rev. Lett. 2003. Vol. 90. P. 160403.

[4] Rossi F., Opat G.I. // J. Phys. 1992. Vol. D25. P. 1349.

[5] Sacha G.M., Verdaguer A., Martinez J., Saenz J.J., Ogletree D.F., Salmeron M. // Appl. Phys. Lett. 2005. Vol. 86. P. 123101.

[6] Sacha G.M., Sahagun E., Saenz J.J. // J. Appl. Phys. 2007. Vol. 101. P. 024310.

[7] Дедков Г.В., Канаметов А.А., Дедкова Е.Г. // ЖТФ. 2009. Т. 79. Вып. 12. С. 79.

[8] Chen F., Mohideen U. // J. Phys. A: Math. Gen. 2006. Vol. 39. P. 6223.

[9] Batra R.C., Porfiri M., Spinello D. // J. Sound Vib. 2008. Vol. 309. N 3. P. 600.

[10] Hu Y.J., Yang J., Kitipornchai S. // J. Sound Vib. 2013. Vol. 332. N 15. P. 3821.

[11] Stipe B.C., Mamin H.J., Stowe T.D., Kenny T.W., Rugar D. // Phys. Rev. Lett. 2001. Vol. 87. P. 096801.

[12] Chumak A.A., Milonni P.W., Berman G.P. // Phys. Rev. 2004. Vol. B70. P. 085407.

[13] Kuehn S., Loring R.F., Marohn J. // Phys. Rev. Lett. 2006. Vol. 96. P. 156103.

[14] Kisiel M. et al. // Nature Mat. 2011. Vol. 10. P. 119.

[15] Volokitin A.I., Persson B.N.J. // Rev. Mod. Phys. 2007. Vol. 79. P. 1291.

[16] Дедков Г.В., Кясов А.А. // ФТТ. 2002. Т. 44. Вып. 10. С. 1729.

[17] Дедков Г.В., Канаметов А.А. // Письма в ЖТФ. 2010. Т. 36. Вып. 6. С. 1.

[18] Силадьи М. // Электронная и ионная оптика. М.: Мир, 1990. $638 \mathrm{c}$ 